\title{
ESPÉCIES VEGETAIS DOMINANTES DO ARQUIPÉLAGO DE FERNANDO DE NORONHA: GRUPOS ECOLÓGICOS E REPARTIÇÃO ESPACIAL ${ }^{1}$
}

\author{
Mateus Batistella ${ }^{2}$
}

Recebido em 25/5/95. Aceito em 2/10/96.

RESUMO - (Espécies vegetais dominantes do arquipélago de Fernando de Noronha: grupos ecológicos e repartição espacial). São relacionadas as 60 principais espécies vegetais dominantes do Arquipélago de Fernando de Noronha, no Nordeste brasileiro: 33 herbáceas, 12 lenhosas baixas ( $<2$ metros) e 15 lenhosas altas ( $>2$ metros). A partir de tratamentos numéricos analíticos, as informaçōes mútuas para cada espécie em relação a variáveis ambientais evidencia grupos ecológicos distintos, caracterizados por seus padrões de repartição espacial. As plantas com padrão de repartição espacial definido dividem-se em espécies ubiquistas, de sotavento, de barlavento, costeiras, de áreas interiores, de áreas florestadas e de ocorrência isolada. As plantas com padrão pouco definido são classificadas mais por sua função na paisagem que pelo modo de repartição espacial nas ilhas e ilhotas. São as jitiranas, as invasoras e as espécies plantadas ou ornamentais.

Palavras-chave: arquipélago de Fernando de Noronha, espécies vegetais dominantes, informação mútua, padrōes de repartição espacial.

\begin{abstract}
Dominant plant species of the Fernando de Noronha archipelago: ecological groups and spatial distribution).This paper relates the 60 main dominant plant species of the Fernando de Noronha Archipelago, on the Brazilian Northeast. Using analytical numeric treatments, the mutual information indexes were calculated for each species regarding to environmental variables. The results allowed the identification of seven groups of plants with defined patterns and three groups with less defined patterns of spatial distribution. The plants with defined patterns were divided into ubiquitous plants, species of windward and leeward coastal zones, interior and forested areas and isolated plants. The species with less defined spatial patterns were classified by their function on the islands landscape.
\end{abstract}

Key-words: Fernando de Noronha archipelago, dominant plant species, mutual information, spatial patterns.

Parte da dissertação de Mestrado. Depto de Ecologia Geral. Instituto de Biociências. USP

2 Pesquisador da Embrapa/NMA. Av. Dr. Júlio Soares de Arruda, 803. C. P. 491. CEP 13088-300

Campinas - SP. E-mail: mb@nma.embrapa.br 


\section{Introdução}

Desde seu descobrimento, em 1503, o arquipélago de Fernando de Noronha tem despertado muitos interesses, seja por seu posicionamento estratégico, ou pelas características singulares de seus recursos naturais.

Durante quase cinco séculos de ocupação humana, esse conjunto de pequenas ilhas esteve vinculado a várias instâncias administrativas. Cada período teve seu reflexo particular sobre o meio ambiente, causando maior ou menor impacto sobre a composição e estrutura da vegetação. A descontinuidade de gestões dificultou ainda mais a tomada de decisões relativas ao manejo dos recursos já ameaçados do arquipélago, principalmente no que se refere a seus ecossistemas terrestres.

Nestes últimos anos, o Brasil redescobriu o "paraíso perdido do Atlântico", palco de inúmeras reportagens, minisséries e até novelas. Esse fenômeno estimulou a liberação total das visitas e dos meios de acesso, a multiplicação de pousadas improvisadas, a construção de casas e infraestrutura urbana, entre outras atividades humanas.

As características ímpares deste sistema insular e a urgência de ordenamento das atividades de desenvolvimento e preservação ecológica exigem a caracterização dos principais condicionantes da paisagem noronhense (Batistella 1993).

As primeiras visitas a campo confirmaram a pertinência de uma hipótese principal sobre a existência de fatores ecológicos determinantes na gênese e evolução das paisagens e na repartição espacial das espécies vegetais dominantes do arquipélago.

Mesmo ao observador menos atento, é possível verificar que as pequenas ilhas de Fernando de Noronha oferecem uma fisionomia vegetal bastante monótona, predominada por plantas herbáceas, muitas vezes em formações quase monoespecíficas. Ao contrário, nas ilhas de maiores dimensões e substrato variável, a vegetação assume diversas fisionomias, compostas por um maior número de espécies.

Em todas essas situações, a visível repetição e expressiva cobertura de certas plantas permitem a identificação de espécies que dominam a fisionomia vegetal. A ocorrência dessas espécies imprime características singulares na paisagem, seja pela estatura, cor e textura, seja pela ação que exercem sobre o habitat, a partir de seu recobrimento e variabilidade sazonal, como previsto em Crawley (1986).

Portanto, além de listar as espécies vegetais dominantes identificadas, o objetivo do trabalho foi relacionar a ocorrência dessas plantas com os descritores ambientais amostrados, através de tratamentos numéricos analíticos baseados em cálculos de natureza hipergeométrica.

\section{Material e métodos}

\section{Área de estudo}

O arquipélago de Fernando de Noronha está localizado a 350'24" de Latitude Sul e $32^{\circ} 24^{\prime} 48^{\prime \prime}$ de Longitude Oeste de Greenwich (coordenadas do Posto Meteorológico), a $345 \mathrm{Km}$ do Cabo de São Roque no Rio Grande do Norte, seu ponto mais 
próximo em terra firme. Sua menor distância da costa africana (Libéria) é, aproximadamente, de $2600 \mathrm{Km}$ (Fig. 1).



Figura 1. Localização do arquipélago de Fernando de Noronha (Modificado de Soares 1944).

Possuindo 17 ilhas e ilhotas, além de rochedos isolados, em um total aproximado de 1926 ha de extensão, o arquipélago é o que restou do topo emerso de um edifício vulcânico localizado a cerca de $4000 \mathrm{~m}$ de profundidade, parte de uma ramificação da Dorsal Médio Atlântica em direção à Costa Brasileira (Almeida 1958).

A ilha Fernando de Noronha é o único ambiente insular oceânico brasileiro constantemente habitado há mais de quatro séculos. Possui 1766 ha de extensão, maior dimensão de $10 \mathrm{Km}$ orientada no eixo NNE - SSW e larguras máximas de 2,0 a 3,3 $\mathrm{Km}$. Sua principal elevação é o Morro do Pico, com $323 \mathrm{~m}$ de altitude. 
O clima é do tipo Awi de Köppen, porém marcado pelo domínio oceânico. A média anual das temperaturas é de $27^{\circ} \mathrm{C}$, com amplitude térmica anual menor que $2^{\circ} \mathrm{C}$. $\mathrm{O}$ total das precipitações anuais situa-se em torno de $1400 \mathrm{~mm}$, porém com grande variabilidade interanual, acompanhando a tendência de toda a faixa tropical que abrange o Nordeste continental. São evidentes duas estações bem marcadas, indo o período úmido de março a julho. Um importante fator do clima de Fernando de Noronha é a intensa e constante ventilação. Os alíseos de ESE atingem principalmente a fachada barlavento (Araújo 1981), provocando cosequências perceptíveis sobre o meio e a vegetação.

Acompanhando o regime pluviométrico de regiões sub-úmidas e semi-áridas, os pequenos rios e riachos são torrenciais, secando todos os anos na época da estiagem, salvo raras exceções (BRASIL 1981). A morfologia noronhense atual é decorrência das ações climáticas, marinhas e fluviais sobre o embasamento geológico de origem vulcânica, variando de acordo com a formação de cada ilha, desde platôs arenosos ou basálticos até altos rochedos escarpados.

A diversidade pedológica do arquipélago é menor do que a diferenciação de materiais de seu substrato autorizaria aguardar. As principais associações pedológicas, geralmente de alta fertilidade, são predominadas por cambissolos e solos litólicos eutróficos, seguidas de ocorrências de areias calcárias marinhas e solo salino indiscriminado (Mantovani et al. 1992).

Em termos biológicos, o arquipélago é rico em endemismos, porém bastante pobre, tanto em relação à sua flora (Silva \& Felfini 1986), quanto à colonização por espécies faunísticas (Miranda 1991). A distância no Atlântico, o isolamento do continente mais próximo em termos de correntes marítimas e ventos dominantes, a pequena extensão territorial, o término relativamente recente das manifestações vulcânicas e o clima semi-árido estão entre as principais causas dessa baixa riqueza específica.

Recursos disponíveis e procedimentos adotados

No início do trabalho foi realizada uma extensa reunião dos materiais bibliográficos, cartográficos e fotográficos disponíveis sobre Fernando de Noronha. Foram catalogadas as principais referências, consultadas várias instituições que desenvolveram pesquisas no arquipélago e compilada toda a base cartográfica disponível nas escalas 1:10.000 a 1:50.000.

Os limites fotointerpretados para a fisionomia da vegetação, demarcados sobre pares de fotos aéreas pancromáticas na escala 1:10.000 (FORÇA AÉREA BRASILEIRA 1986), permitiram o reconhecimento preliminar da heterogeneidade macroecológica das terras emersas. Partindo da hipótese inicial de que as espécies vegetais do arquipélago são sensíveis a esta heterogeneidade, optou-se pela estratégia de amostragem estratificada aleatória para o trabalho de campo (Legendre \& Legendre 1983).

Foram definidos 56 estratos, a partir da combinação das classes de quatro variáveis passíveis de identificação nas fotografias aéreas: grau de artificialização, 
recobrimento do solo, heterogeneidade da estrutura horizontal da vegetação e influência do vento sobre a vegetação e o meio (AMERICAN SOCIETY OF PHOTOGRAMMETRY 1960; Godron et al. 1968; Anderson \& Verstappen 1982).

Para cada um dos estratos, foram sorteados cerca de 10 levantamentos de campo, de acordo com o plano de amostragem previamente elaborado. A variação do número de levantamentos por estrato, em torno desta média, esteve relacionada aos problemas de acesso ao local determinado ou à necessidade de maior ou menor esforço amostral. Essas decisões eram tomadas ao longo das etapas de campo, a partir da análise da heterogeneidade dos estratos, conforme previsto em Frontier (1983).

Os levantamentos foram realizados durante a estação chuvosa, para facilitar a identificação das espécies vegetais dominantes, geralmente caducifólias e com fenologia condicionada pela sazonalidade local.

A ficha de reconhecimento foi inspirada em trabalhos análogos (Jurdant et al. 1977; Duranton et al. 1982) e continha 55 descritores qualitativos e quantitativos sobre o local de levantamento (unidades morfopedológicas, superfície aproximada, data, hora etc.); o meio físico (situação topográfica, pendente, exposição, cobertura da superfície, compacidade do solo, morfogênese, ação do vento, influência oceânica etc.); a vegetação (categoria dos vegetais dominantes, espécies dominantes, formação vegetal, grau de abertura dos estratos, fitodinâmica etc.); e a influência antrópica sobre a vegetação e o meio (acomodação da vegetação por pastejo e pisoteio, atividade agrícola, grau de artificialização etc.). A ficha completa está publicada em Batistella (1993).

Após a localização do ponto amostral, os levantamentos foram efetuados em porções limitadas de território, onde as condições ecológicas são homogêneas e a vegetação, em geral, uniforme, definindo as estações ecológicas passíveis de serem amostradas (Godron et al. 1968). A área de levantamento variou em função das dimensões do polígono demarcado pela fotointerpretação. As estações de coleta foram fotografadas e exemplares vegetais herborizados para identificação. As fotos receberam o número do levantamento e foram posteriormente exploradas como um registro das condições ecológicas apresentadas pelo ponto de prospecção. No total, foram amostradas 618 estações ecológicas, 558 das quais com presença de espécies vegetais.

Foram consideradas dominantes as plantas herbáceas, lenhosas baixas $(<2 \mathrm{~m}) \mathrm{e}$ altas $(>2 \mathrm{~m})$ que mais contribuem visualmente à fisionomia da vegetação. São espécies cujas projeções horizontais do aparelho aéreo de seus indivíduos geralmente representam mais de $20 \%$ do recobrimento de todo o estrato vertical a que pertencem. A utilização de classes pré-definidas reduziu o erro subjetivo na estimação da cobertura, tornando o procedimento rápido e eficiente, como previsto em Conant et al. (1983).

A identificação das plantas beneficiou-se de trabalhos anteriores sobre 0 arquipélago, particularmente aqueles que incluíam uma lista florística (Ridley 1888; HIDROSERVICE 1986; Duranton \& Launois-Luong 1988).

Foram calculados quatro parâmetros numéricos a partir dos dados coletados: frequência absoluta (FA), frequência relativa (FR), frequência corrigida (FC) e informação mútua (IM). A tabela 1 resume, segundo Guillerm (1971), os três tipos de perfis ecológicos baseados em cálculo de freqüências. 
Tabela 1. Perfis ecológicos de uma espécie E por um descritor L.

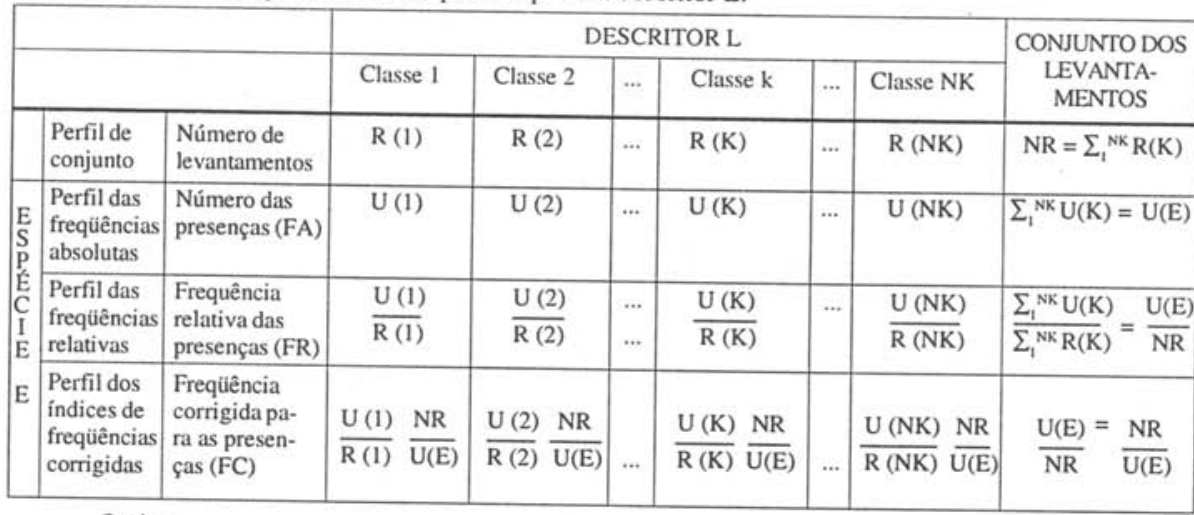

Onde:

- $\mathrm{NK}=$ número de classes distintas pelo descritor $\mathrm{L}$

- $\mathrm{U}(\mathrm{K})$ = número de levantamentos da classe $\mathrm{K}$, onde a espécie $\mathrm{E}$ está presente

- $R(K)=$ número de levantamentos da classe $K$

- $\mathrm{U}(\mathrm{E})=$ número total de levantamentos onde a espécie $\mathrm{E}$ está presente

- NR = número total de levantamentos com espécies vegetais presentes

Com a utilização de programas escritos em linguagem APL (Daget \& Godron 1982), a informação mútua para uma espécie e um fator ecológico forneceu uma estimativa do valor indicador da espécie para o conjunto das classes deste fator, em função das outras espécies e dos outros fatores amostrados (Guinochet 1973).

Para o cálculo da informação mútua, foram utilizados os conjuntos de dados referentes aos descritores amostrados nas campanhas de levantamentos e à lista das espécies vegetais dominantes identificadas no arquipélago. Em termos numéricos, ela é definida pela quantidade de informação IM(i;k), obtida no perfil ecológico de uma espécie k por um fator i (Daget \& Godron 1982). Expressa-se por uma diferença de entropia:

$\operatorname{IM}(\mathrm{i} ; \mathrm{k})=\mathrm{h}(\mathrm{i})-\mathrm{H}(\mathrm{i} / \mathrm{k})$, onde:

- h(i) é a entropia do fator i ( a priori);

- H(i/k) é a entropia do fator i, a partir da presença ou ausência da espécie $\mathrm{k}$ (a posteriori).

O resultado permitiu estimar a ligação de cada espécie vegetal dominante identificada no arquipélago em relação aos descritores da ficha de reconhecimento. Em outras palavras, foi mensurada a probabilidade de co-ocorrência entre as espécies e cada uma das classes das variáveis amostradas no campo.

\section{Resultados e discussão}

No total, foram identificadas 60 espécies vegetais dominantes em Fernando de Noronha (tab. 2). Não foram incluídas as plantas introduzidas para fins agrícolas, ge- 
ralmente de ocorrência restrita a pequenas áreas isoladas. Por outro lado, foram mantidas as espécies invasoras e ornamentais, pois além de apresentarem importantes expressões espaciais, algumas delas são hoje consideradas subespontâneas no arquipélago.

Tabela 2. Freqüência absoluta (FA) e relativa (FR), estrato vertical (EST) e padrão de repartição espacial (R) das espécies vegetais dominantes identificadas no arquipélago de Fernando de Noronha.

\begin{tabular}{|c|c|c|c|c|c|}
\hline ESPÉCIES DOMINANTES & FAMILIA & FA & $\mathrm{FR}(\%)$ & EST. & $\mathrm{R}$ \\
\hline Senna obtusifolia (L.) Irwin \& Barneby & Fabaceae & 192 & 34,41 & $\mathrm{H}$ & In \\
\hline Sapium sceleratum Ridl. & Euphorbiaceae & 157 & 28,14 & LA & $\mathrm{U}$ \\
\hline Jatropha curcas L. & Euphorbiaceae & 119 & 21,33 & LB & $\mathrm{U}$ \\
\hline Capparis cynophallophora $\mathrm{L}$. & Capparaceae & 109 & 19,53 & LA & $\mathrm{U}$ \\
\hline Lantana camara $\mathrm{L}$. & Verbenaceae & 109 & 19,53 & LB & $\mathrm{U}$ \\
\hline Ipomoea purpurea (L.) Roth & Convolvulaceae & 85 & 15,23 & $\mathrm{H}$ & $\mathbf{J}$ \\
\hline Leucaena leucocephala (Lam.) De Wit & Fabaceae & 71 & 12,72 & LA & $\mathrm{U}$ \\
\hline Capparis flexuosa $\mathrm{L}$. & Capparaceae & 67 & 12,01 & LB & $\mathrm{F}$ \\
\hline Oxalis insipida $\mathrm{St}-\mathrm{Hil}$. & Oxalidaceae & 61 & 10,93 & LB & $\mathrm{C}$ \\
\hline Spondias lutea $\mathrm{L}$. & Anacardiaceae & 56 & 10,04 & LA & $\mathrm{P}$ \\
\hline Waltheria indica $\mathrm{L}$. & Sterculiaceae & 55 & 9,86 & $\mathrm{H}$ & $\mathrm{U}$ \\
\hline Erythrina velutina Willd. f. aurantiaca & Fabaceae & 52 & 9,32 & LA & $\mathrm{U}$ \\
\hline Desmanthus virgatus (L.) Willd. & Fabaceae & 50 & 8,96 & $\mathrm{H}$ & In \\
\hline Merremia aegyptia (L.) Urban & Convolvulaceae & 50 & 8,96 & $\mathrm{H}$ & $\mathbf{J}$ \\
\hline Guettarda angelica Mart. ex Muell. Arg. & Rubiaceae & 46 & 8,24 & LA & $\mathrm{F}$ \\
\hline Bumelia sartorum Mart. & Sapotaceae & 44 & 7,88 & LA & $\mathrm{U}$ \\
\hline Paspalum paniculatum $\mathrm{L}$. & Poaceae & 42 & 7,53 & $\mathrm{H}$ & B \\
\hline Momordica charantia L. & Cucurbitaceae & 39 & 6,99 & $\mathrm{H}$ & $\mathbf{J}$ \\
\hline Acacia farnesiana Willd. & Fabaceae & 38 & 6,81 & LB & I \\
\hline Cynodon dactylon (L.) Pers. & Poaceae & 37 & 6,63 & $\mathrm{H}$ & In \\
\hline Jasminum fluminensis Vell. & Oleaceae & 35 & 6,27 & $\mathrm{H}$ & $\mathrm{S}$ \\
\hline Tabebuia roseo-alba (Ridl.) Sandwith & Bignoniaceae & 35 & 6,27 & LA & $\mathrm{F}$ \\
\hline Cereus insularis Hemsl. & Cactaceae & 34 & 6,09 & LB & $\mathrm{C}$ \\
\hline Cyperus rotundus $\mathrm{L}$. & Cyperaceae & 34 & 6,09 & $\mathrm{H}$ & In \\
\hline Jacquinia armillaris Jacq. & Theophrastaceae & 30 & 5,38 & LB & B \\
\hline Jatropha gossypifolia L. & Euphorbiaceae & 30 & 5,38 & LB & I \\
\hline Calopogonium mucunoides Desv. & Fabaceae & 29 & 5,20 & $\mathrm{H}$ & $\mathrm{J}$ \\
\hline Vitis sicyoides Miq. & Vitaceae & 29 & 5,20 & $\mathrm{H}$ & $\mathrm{U}$ \\
\hline Cyperus articulatus L. & Cyperaceae & 27 & 4,84 & $\mathrm{H}$ & B \\
\hline Jatropha mollissima Baill. & Euphorbiaceae & 27 & 4,84 & LB & I \\
\hline Brachiaria plantaginea (Link) Hitchc. & Poaceae & 25 & 4,48 & $\mathrm{H}$ & In \\
\hline Ficus cf. catappifolia Kunth \& Bouché ex Kunth & Moraceae & 25 & 4,48 & LA & $\mathrm{U}$ \\
\hline Terminalia catappa $\mathrm{L}$. & Combretaceae & 24 & 4,30 & LA & $\mathrm{P}$ \\
\hline Philoxerus portulacoides St-Hil. & Amaranthaceae & 22 & 3,94 & $\mathrm{H}$ & B \\
\hline Croton odoratus Ridl. & Euphorbiaceae & 19 & 3,41 & $\mathrm{H}$ & In \\
\hline Ipomoea pes-caprae (L.) R. Br. & Convolvulaceae & 19 & 3,41 & $\mathrm{H}$ & $\mathrm{C}$ \\
\hline Macroptilium gracile (Poepp. ex Benth.) Urban & Fabaceae & 18 & 3,23 & $\mathrm{H}$ & $\mathrm{J}$ \\
\hline Amaranthus spinosus L. & Amaranthaceae & 17 & 3,05 & $\mathrm{H}$ & In \\
\hline Eleusine indica (L.) Gaertn. & Poaceae & 17 & 3,05 & $\mathrm{H}$ & In \\
\hline Palicourea insularis Ridl. & Rubiaceae & 16 & 2,87 & LB & $\mathrm{B}$ \\
\hline Abrus precatorius L. & Fabaceae & 15 & 2,69 & $\mathrm{H}$ & S \\
\hline Panicum latifolium Hamilt. & Poaceae & 13 & 2,33 & $\mathrm{H}$ & In \\
\hline Calotropis procera (Ait.) R. Br. & Asclepiadaceae & 12 & 2,15 & LB & Is \\
\hline Euphorbia tirucalli $\mathrm{L}$. & Euphorbiaceae & 11 & 1,97 & LB & $\mathrm{P}$ \\
\hline Fimbristylis spathacea Roth & Cyperaceae & 11 & 1,97 & $\mathrm{H}$ & B \\
\hline Licania tomentosa (Benth.) Fritsch & Chrysobalanaceae & 9 & 1,61 & LA & $\mathrm{P}$ \\
\hline Echinochloa colonum (L.) Link & Poaceae & 7 & 1,25 & $\mathrm{H}$ & In \\
\hline Panicum maximum Jacq. & Poaceae & 7 & 1,25 & $\mathrm{H}$ & In \\
\hline Indigofera lespedezioides H.B.K. & Fabaceae & 6 & 1,08 & $\mathrm{H}$ & I \\
\hline
\end{tabular}


Boerhaavia repens $\mathrm{L}$.

Malachra fasciata Jacq.

Manihot tripartita Muell. Arg.

Cleome diffusa Banks ex DC.

Cnidosculus urens (L.) Arthur

Delonix regia (Boj. ex Hook.) Raf.

Pistia stratiotes $\mathrm{L}$.

Anadenanthera macrocarpa (Benth.) Brenan.

Laguncularia racemosa Gaertn.

Phyllanthus niruri L.

Turnera ulmifolia $\mathrm{L}$.
Nyctaginaceae

Malvaceae

Euphorbiaceae

Capparaceae

Euphorbiaceae

Fabaceae

Araceae

Fabaceae

Combretaceae

Euphorbiaceae

Turneraceae
0,90

0.90

0.90

0,54

0,54

0,54

0,36

0,18

0,18

0,18

0,18
H W

H W

LA I

H I

H N

LA Is

$\mathrm{H}$ Is

LA P

LA Is

$\mathrm{H}$ Is

$\mathrm{H}$ Is

LEGENDA: $F A=$ frequência absoluta; $F R=$ frequência relativa; $E S T=$ estrato vertical predominante; $H$ = herbáceo; $\mathrm{LB}=$ lenhoso baixo $\mathrm{LA}=$ lenhoso alto; $\mathrm{R}=$ repartição espacial; Padrōes definidos: $\mathrm{U}=$ ubiquistas; $\mathrm{S}=$ sotavento; $\mathrm{B}=$ barlavento; $\mathrm{C}=$ áreas costeiras; $\mathrm{F}$ : áreas florestadas pouco artificializadas; $\mathrm{I}=$ áreas interiores; Is = ocorrência isolada; Padrões pouco definidos: $\mathrm{In}=$ invasoras; $\mathrm{J}=$ jitiranas; $\mathrm{P}=$ espécies plantadas ou ornamentais.

Foram distintos sete padrões definidos de repartição espacial: as espécies ubiquistas, de sotavento, de barlavento, de áreas costeiras, de áreas interiores, de áreas florestadas pouco artificializadas e de ocorrência isolada. Também foram considerados outros três grupos de espécies vegetais com padrões pouco definidos de repartição espacial: as jitiranas, as invasoras e as espécies plantadas ou ornamentais (Tab. 3).

Tabela 3. Padrões de repartição espacial e estrato vertical das espécies vegetais dominantes do arquipélago de Fernando de Noronha.

\begin{tabular}{|c|c|c|c|c|}
\hline $\begin{array}{l}\text { Estratos } \\
\text { Padrōes definidos }\end{array}$ & Herbáceas & Lenhosas Baixas & Lenhosas Altas & Total \\
\hline Ubiquistas & 2 & 2 & 6 & 10 \\
\hline Sotavento & 2 & . & - & 2 \\
\hline Barlavento & 4 & 2 & - & 6 \\
\hline Costeiras & 1 & 2 & - & 3 \\
\hline Interiores & 1 & 3 & . & 4 \\
\hline Florestas & - & I & 2 & 3 \\
\hline Isoladas & 7 & 1 & 3 & 11 \\
\hline Sub-total & 17 & 11 & 11 & 39 \\
\hline \multicolumn{5}{|c|}{$\underline{\text { Padrões pouco definidos }}$} \\
\hline Jitiranas & 5 & - & - & 5 \\
\hline Invasoras & 11 & - & - & 11 \\
\hline Plantadas & - & 1 & 4 & 5 \\
\hline Sub-total & 16 & 1 & 4 & 21 \\
\hline Total & 33 & 12 & 15 & 60 \\
\hline
\end{tabular}

Padrões definidos de repartição espacial

As espécies vegetais ubiquistas não apresentam sua repartição espacial associada a um fator ecológico particular ou local determinado. São dominantes tanto na ilha principal como nas ilhotas, em maior ou menor freqüência relativa dos levantamentos 
executados no campo. Entre elas estão Sapium sceleratum, a árvore mais frequente do arquipélago, e Erythrina velutina f. aurantiaca, uma das principais espécies da comunidade arbórea de Fernando de Noronha.

A tabela 4 indica a afinidade de algumas espécies vegetais aos ambientes condicionados pela intensidade dos alíseos. Embora com valores medianos de informação mútua, nota-se a maior probabilidade de ocorrência de Abrus precatorius e Jasminum fluminensis associada à classe de fraca acomodação da vegetação pelo vento no arquipélago.

Tabela 4. Informação mútua de algumas espécies vegetais dominantes em relação à acomodação da vegetação pelo vento no arquipélago de Fernando de Noronha (FA = Frequêencia Absoluta; $I M=$ Informação Mútua, $\mathrm{FC}=$ Frequência Corrigida).

\begin{tabular}{|c|c|c|c|c|c|c|c|}
\hline \multicolumn{2}{|c|}{$\mathrm{N}^{\circ}$ de Levantamentos } & $\begin{array}{c}\text { Nula } \\
3\end{array}$ & $\begin{array}{c}\text { Fraca } \\
133\end{array}$ & $\begin{array}{c}\text { Média } \\
237\end{array}$ & $\begin{array}{c}\text { Forte } \\
91\end{array}$ & $\begin{array}{c}\text { M.Forte } \\
118\end{array}$ & $\begin{array}{c}\text { Total } \\
618\end{array}$ \\
\hline FA & $\mathrm{IM}$ & FC & FC & FC & $\mathrm{FC}$ & FC & \\
\hline 67 & .21 & 615 & 381 & 34 & 0 & 0 & Capparis flexuosa \\
\hline 46 & .15 & 0 & 394 & 10 & 74 & 0 & Guettarda angelica \\
\hline 35 & .11 & 589 & 345 & 13 & 116 & 0 & Tabebuia roseo-alba \\
\hline 42 & .17 & 0 & 11 & 0 & 49 & 474 & Paspalum paniculatum \\
\hline 22 & .08 & 0 & 0 & 31 & 93 & 381 & Philoxerus portulacoides \\
\hline 27 & .08 & 0 & 0 & 25 & 377 & 175 & Cyperus articulatus \\
\hline 30 & .07 & 0 & 217 & 8 & 91 & 192 & Jacquinia armillaris \\
\hline 11 & .05 & 0 & 0 & 21 & 185 & 333 & Fimbristylis spathacea \\
\hline 35 & .10 & 0 & 345 & 26 & 97 & 0 & Jasminum fluminensis \\
\hline 15 & .07 & 0 & 403 & 30 & 0 & 0 & Abrus precatorius \\
\hline
\end{tabular}

As espécies de barlavento são características da fachada sul do arquipélago, pertencendo geralmente a formações vegetais simples de herbáceas ou complexas de herbáceas e lenhosas baixas. Nestes locais, a intensa ventilação durante quase todo o ano provoca sinais visíveis na estrutura e fisionomia da vegetação, direcionando os ramos, condicionando a existência de plantas com folhas coriáceas e reduzindo a altura dos estratos lenhosos em relação aos locais mais abrigados dos alíseos. A tabela 4 confirma a co-ocorrência de algumas destas espécies dominantes com as classes de maior acomodação da vegetação pelo vento. O melhor exemplo é Paspalum paniculatum, dominante em extensas formações herbáceas quase monoespecíficas.

Outras três espécies vegetais ocorrem no arquipélago com expressiva dominância em áreas próximas à costa, tanto na fachada sotavento como barlavento: Cereus insularis (Cactaceae), Ipomoea pes-caprae (Convolvulaceae) e Oxalis insipida (Oxalidaceae). A tabela 5 indica a informação mútua destas e outras espécies em relação à intensidade de penetração da bruma marinha nos biótopos terrestres, através das gotículas de água salgada trazidas pelos ventos.

Na mesma tabela 5 estão relacionadas três lenhosas baixas e uma herbácea pertencentes a duas famílias que apresentam ocorrência predominante em áreas distanciadas da costa, porém sem relação direta a algum padrão de ocorrência isolada. 
São elas Acacia farnesiana e Indigofera lespedezioides (Fabaceae), Jatropha gossypifolia e Jatropha molissima (Euphorbiaceae). Todas ocorrem como dominantes em formações vegetais com influência nula ou muito fraca da bruma marinha.

Tabela 5. Informação mútua de algumas espécies vegetais dominantes em relação à influência oceânica na vegetação do arquipélago de Fernando de Noronha (FA = Frequência Absoluta; IM = Informação Mútua; $\mathrm{FC}=$ Frequência Corrigida).

\begin{tabular}{|c|c|c|c|c|c|c|c|}
\hline \multicolumn{2}{|c|}{$\begin{array}{c}\text { Classes } \\
\mathrm{N}^{\circ} \text { de Levantamentos } \\
\end{array}$} & \multirow{2}{*}{$\begin{array}{c}\begin{array}{c}\text { Nula } \\
160\end{array} \\
\text { FC }\end{array}$} & \multirow{2}{*}{$\begin{array}{c}\begin{array}{c}\text { Fraca } \\
164\end{array} \\
\text { FC }\end{array}$} & \multirow{2}{*}{$\begin{array}{c}\text { Média } \\
64 \\
\text { FC }\end{array}$} & \multirow{2}{*}{$\begin{array}{c}\begin{array}{c}\text { Forte } \\
69\end{array} \\
\text { FC } \\
\end{array}$} & \multirow{2}{*}{$\begin{array}{c}\begin{array}{c}\text { M.Forte } \\
161\end{array} \\
\text { FC }\end{array}$} & \multirow[t]{2}{*}{$\begin{array}{c}\text { Total } \\
618 \\
\end{array}$} \\
\hline FA & $\mathrm{IM}$ & & & & & & \\
\hline 42 & .13 & 0 & 0 & 46 & 171 & 292 & Paspalum paniculatum \\
\hline 22 & .10 & 0 & 0 & 0 & 0 & 384 & Philoxerus portulacoides \\
\hline 67 & .12 & 121 & 214 & 86 & 27 & 0 & Capparis flexuosa \\
\hline 34 & .08 & 0 & 144 & 0 & 237 & 135 & Cereus insularis \\
\hline 61 & .08 & 32 & 62 & 237 & 220 & 101 & Oxalis insipida \\
\hline 19 & .07 & 0 & 20 & 0 & 236 & 263 & Ipomoea pes-caprae \\
\hline 38 & .08 & 163 & 178 & 51 & 47 & 0 & Acacia farnesiana \\
\hline 27 & .07 & 243 & 126 & 36 & 0 & 0 & Jatropha mollissima \\
\hline 30 & .05 & 103 & 151 & 129 & 119 & 26 & Jatropha gossypifolia \\
\hline 6 & .04 & 258 & 126 & 0 & 0 & 0 & Indigofera lespedezioides \\
\hline
\end{tabular}

Existem também setores onde a vegetação florestal está relativamente bem preservada. Localizam-se principalmente na porção sudoeste da ilha maior. Representados por uma espécie de lenhosa menor que $2 \mathrm{~m}$ e por duas lenhosas altas, a distribuição dos levantamentos entre as classes da variável "grau de artificialização" para Capparis flexuosa, Guettarda angelica e Tabebuia roseo-alba está relacionada na tabela 6 .

Tabela 6. Informação mútua de algumas espécies vegetais dominantes em relação ao grau de artificialização da vegetação e do meio do arquipélago de Fernando de Noronha (FA = Frequêência Absoluta; $I \mathrm{M}=$ Informação Mútua; FC = Frequência Corrigida).

\begin{tabular}{rrrrrrrrrl}
\hline \multicolumn{2}{c}{$\begin{array}{c}\text { Classes } \\
\text { N de Levantamentos }\end{array}$} & $\begin{array}{r}\text { Nulo } \\
125\end{array}$ & $\begin{array}{c}\text { M.Fraco } \\
103\end{array}$ & $\begin{array}{c}\text { Fraco } \\
98\end{array}$ & $\begin{array}{c}\text { Médio } \\
84\end{array}$ & $\begin{array}{r}\text { Forte } \\
41\end{array}$ & $\begin{array}{c}\text { M.Forte } \\
90\end{array}$ & $\begin{array}{c}\text { Máx. } \\
77\end{array}$ & $\begin{array}{c}\text { Total } \\
618\end{array}$ \\
\hline FA & IM & FC & FC & FC & FC & FC & FC & FC & \\
\hline 192 & .31 & 5 & 28 & 62 & 222 & 204 & 111 & 196 & Senna obrusifolia \\
109 & .22 & 0 & 44 & 249 & 236 & 138 & 57 & 29 & Lantana camara \\
37 & .17 & 0 & 0 & 0 & 40 & 81 & 111 & 586 & Cynodon dactylon \\
34 & .13 & 0 & 0 & 19 & 65 & 222 & 384 & 142 & Cyperus rotundus \\
67 & .21 & 37 & 403 & 141 & 22 & 0 & 0 & 0 & Capparis flexuosa \\
46 & .14 & 54 & 365 & 123 & 64 & 0 & 0 & 0 & Guettarda angelica \\
35 & .12 & 71 & 326 & 198 & 0 & 0 & 0 & 0 & Tabebuia roseo-allha \\
56 & .12 & 0 & 75 & 23 & 171 & 188 & 221 & 129 & Spondias lutea \\
24 & .11 & 0 & 25 & 26 & 31 & 63 & 114 & 535 & Terminalia catappa \\
11 & .08 & 0 & 0 & 0 & 0 & 0 & 312 & 438 & Euphorbia tirucalli \\
\hline
\end{tabular}

A tabela 6 também relaciona sete espécies dominantes em áreas com expressivo grau de artificialização, definindo o grupo das invasoras (Senna obtusifolia, Lantana camara, Cynodon dactylon e Cyperus rotundus) e das espécies plantadas ou ornamentais (Spondias lutea, Terminalia catappa e Euphorbia tirucalli). 
O último padrão definido de repartição espacial é representado por onze espécies vegetais dominantes: sete herbáceas, uma lenhosa baixa e três lenhosas altas. Caracterizadas por ocorrências isoladas, a freqüência relativa destas espécies nos levantamentos nunca é maior que $2 \%$ e a área ocupada sempre menor que $0,5 \%$ do arquipélago. Geralmente são fatores ecológicos específicos que condicionam estas expressões espaciais quantitativamente discretas, mas com significados determinados. Entre eles, estão as características do substrato (Cnidosculus urens), o isolamento geográfico em ilhotas (Boerhaavia repens) e a existência de habitats particulares, tais como o mangue de Sueste (Laguncularia racemosa), as dunas (Calotropis procera, Phyllanthus niruri e Turnera ulmifolia), as lagoas (Cleome diffusa), os açudes (Pistia stratiotes), entre outros.

\section{Padrões pouco definidos de repartição espacial}

Cerca de um terço dos vegetais dominantes identificados no arquipélago apresenta padrões pouco definidos de repartição espacial. São 21 espécies, cuja ocorrência está geralmente associada à maior interferência do homem na estrutura e na composição das comunidades vegetais.

Foram distintos três grupos: as jitiranas, as invasoras e as espécies plantadas ou ornamentais. Os dois primeiros são representados por 16 espécies herbáceas no total. As plantas utilizadas na arborização atual ou pretérita dividem-se em quatro lenhosas altas e uma lenhosa baixa.

As jitiranas, como são chamadas pelos ilhéus, são plantas de hábito volúvel ou trepador, que apresentam anualmente um fenômeno fitodinâmico particular, evidenciado pelo brotamento intenso na estação chuvosa . Neste período, recobrem encostas, escalam obstáculos naturais ou artificiais, tais como cercas, entulhos ou postes e formam densas camadas de matéria verde sobre o solo. São representadas por cinco espécies dominantes, distribuídas entre as famílias Convolvulaceae (Ipomoea purpurea e Merremia aegyptia, Cucurbitaceae (Momordica charantia) e Fabaceae (Calopogonium mucunoides e Macroptilium gracile). Ocorrem principalmente na ilha maior e em algumas porções de outras ilhotas.

As espécies consideradas invasoras, nas estações ecológicas amostradas em Fernando de Noronha, são plantas típicas de estágios iniciais da sucessão vegetal, geralmente relacionados a áreas onde a atividade antrópica foi expressiva. No total, são representadas por onze espécies herbáceas dominantes, divididas entre as famílias Poaceae, Fabaceae, Amaranthaceae, Cyperaceae e Euphorbiaceae. Ocorrendo em populações isoladas ou em comunidades, são grupos de plantas cuja dispersão foi predominantemente influenciada pelo homem. Por esse motivo, são responsáveis pela fisionomia vegetal de grande parte dos planaltos da ilha principal. Não ocorrem em nenhuma outra ilhota, com exceção da Senna obtusifolia, que tem expressões espaciais também nas Ilhas do Meio e Rata.

As espécies plantadas ou ornamentais são quatro lenhosas altas e uma lenhosa baixa, cultivadas pelo homem, com intenção paisagística. Estão representadas pelas 
famílias Anacardiaceae (Spondias lutea), Chrysobalanaceae (Licania tomentosa), Combretaceae (Terminalia catappa), Euphorbiaceae (Euphorbia tirucalli) e Fabaceae (Anadenanthera macrocarpa). Ocorrem como dominantes apenas na Ilha Fernando de Noronha, em padrões pouco definidos de repartição espacial. Porém, estão necessariamente relacionadas a setores bastante artificializados.

Estudos complementares com a finalidade de expressar cartograficamente a ocorrência das espécies dominantes também foram realizados e estão disponíveis no Núcleo de Monitoramento Ambiental (Embrapa/NMA). O acesso a esses dados pode ser feito pela rede Internet, através do endereço http://www.nma.embrapa.br/.

\section{Conclusões}

Os métodos utilizados neste trabalho permitiram a divisão das espécies vegetais dominantes de Fernando de Noronha em grupos ecológicos de diferente repartição espacial.

Os procedimentos adotados foram adequados para a identificação das espécies dominantes, principalmente devido à relativa pobreza florística e à facilidade de reconhecimento das plantas mais representativas na formação vegetal, em termos de recobrimento do substrato.

As relações de co-ocorrência das espécies com os descritores ambientais evidenciaram os principais grupos ecológicos existentes no Arquipélago. Esses resultados foram consequência da conciliação de uma estratégia de amostragem estratificada aleatória, da coleta de dados no campo e dos tratamentos numéricos analíticos.

Cerca de dois terços das plantas identificadas apresentam padrões definidos de repartição espacial, comprovando a hipótese inicial sobre o determinismo imposto pelos descritores ambientais amostrados.

Os resultados obtidos representam mais uma contribuição ao conhecimento e manejo da vegetação do arquipélago, principal representante dos ecossistemas insulares brasileiros.

\section{Agradecimentos}

O autor reserva agradecimentos ao Dr. Evaristo E. de Miranda, pela orientação do trabalho; ao Dr. José Roberto Miranda, pelo acompanhamento dos tratamentơs numéricos; à Dra. Ana Maria Giulietti, pelas sugestões relacionadas à redação do texto; e a Giselda Person e Taís C. S. Suyama, pela edição e editoração do documento final.

\section{Referências bibliográficas}

Almeida, F.F.M. 1958. Geologia e petrologia do Arquipélago de Fernando de Noronha. Ministério da Agricultura - Div. de Geologia e Mineralogia, Rio de Janeiro. 181p.

AMERICAN SOCIETY OF PHOTOGRAMMETRY. 1960. Manual of aerial photointerpretation. ISPRS, Washington. 349p. 
Anderson, P.S. \& Verstappen, H. T. 1982. Aspectos básicos da fotointerpretação. In: Fundamentos da fotointerpretação. Sociedade Brasileira de Cartografia, Rio de Janeiro. p. 42-86.

Araújo, H.F. 1981. Dados estatísticos e meteorológicos de Fernando de Noronha. Secretaria de Meteorologia - Serviço Regional de Proteção ao Vôo de Recife, Recife. 33p.

Batistella, M. 1993. Cartografia ecológica do Arquipélago de Fernando de Noronha. Dissertação de Mestrado em Ecologia, DEG-IBUSP, São Paulo. 235p.

BRASIL. 1981. Planejamento agrícola energético florestal e hídrico do Território Federal de Fernando de Noronha. Governo do Território Federal de Fernando de Noronha/Governo do Estado de São Paulo - Depto. de Águas e Energia Elétrica, São Paulo. 58p.

Conant, F.; Rogers, P.; Baumgardner, M.; Mckell, C.; Dasmann, R. \& Reining, P. 1983. Resource inventory and baseline study methods for developing countries. AAAS, Washington. 539p.

Crawley, M.J. 1986. The structure of plant communities. In: M.J. Crawley (Ed.), Plant Ecology. Backwell, Oxford. p. 1-50.

Daget, P.; Godron, M. 1982. Analyse fréquentielle de l'écologie des espèces dans les communautés. Masson, Paris. 163p.

Duranton, J.F.; Launois, M.A.; Launois-Luong, M.H. \& Lecoq, M. 1982. Manuel de prospection acridienne en zone tropicale sèche. GERDAT-CIRAD-PRIFAS, Paris. 1487p.

Duranton, J.F. \& Launois-Luong, M.H. 1988. Rapport de mission consultative aupress du CNPDA du 16 juillet au 4 septembre 1987. CIRAD-PRIFAS, Montpellier. 92p.

FORÇA AÉREA BRASILEIRA. 1986. Fotos aéreas do Arquipélago de Fernando de Noronha. Ministério da Aeronáutica, Recife.

Frontier, S. 1983. Stratégies d'échantillonnage en écologie. Masson, Paris. 494p.

Godron, M.; Long, G.; Le Floc'H, E.; Poissonet, J.; Sauvage, C. \& Wacquant, J.P. 1968. Code pour le relevé méthodique de la végétation et du milieu. CNRS, Paris. 292p.

Guillerm, J.L. 1971. Information mutuelle et valeur indicatrice des espèces. Acta Oecol. Plant., v.6: 209 225.

Guinochet, M. 1973. Phytosociologie. Masson, Paris. 227p.

HIDROSERVICE. 1986. Plano diretor de desenvolvimento para o Território Federal de Fernando de Noronha. Hidroservice, São Paulo. 561p.

Jurdant, M.; Belair, J.L.; Gerardin, V. \& Ducruc, J.P. 1977. L'inventaire du capital-nature: méthode de classification et cartographie écologique du territoire. Thorn Press, Quebec. 202p.

Legendre, L. \& Legendre, P. 1983. Échantillonnage et traitement des données. In: S. Frontier, 1983. Stratégies d'échantillonnage en écologie. Masson, Paris. 494p.

Lemée, G. 1978. Précis d'écologie végétale. Masson, Paris. 287p.

Mantovani, L.E.; Fritzsons, E. \& Batistella, M. 1991. Solos de Fernando de Noronha. In: Anais Congresso Brasileiro de Ciência do Solo, $23^{\circ}$. Porto Alegre. p. 263.

Miranda, J.R. 1991. Relatório de pesquisas sobre a ecologia de povoamentos e populaçōes de vertebrados terrestres do Arquipélago de Fernando de Noronha. CNPq, São Paulo. 39p.

Ridley, H.N. 1888. Notes on the botany of Fernando de Noronha. Jour. Linnean Societ. 27:1-95.

Silva, J.R. \& Felfini, J.N. 1986. Relatório preliminar sobre a primeira fase do projeto "Levantamento do potencial natural do Arquipélago de Fernando de Noronha". UnB, Brasília. 12p.

Soares, L.de C. 1944. Território de Fernando de Noronha: síntese geográfica. Boletim geográfico, Rio de Janeiro. p. 1018-1035. 